\title{
Longitudinal collective echoes in coasting particle beams
}

\author{
Ahmed Al-Khateeb, ${ }^{*}$ Oliver Boine-Frankenheim, ${ }^{\dagger}$ Rainer W. Hasse, and Ingo Hofmann \\ Gesellschaft für Schwerionenforschung mbH (GSI), Planckstraße 1, D-64291 Darmstadt, Germany
}

(Received 12 November 2002; published 23 January 2003)

\begin{abstract}
Longitudinal ballistic and collective beam echoes with diffusion effects are investigated theoretically. In the presence of the space-charge impedance, the collective echo amplitude is obtained as a closed form expression. In contrast to the ballistic case, the collective echo amplitude consists of one maximum at time $t_{\text {echo }}$. The echo amplitude grows up and damps down with a rate proportional to the Landau damping rate of space-charge waves. The effect of weak diffusion is found to modify the ballistic and the collective echo amplitudes in the same manner. This effect of diffusion was confirmed using a "noiseless," grid-based simulation code. As a first application the amount of numerical diffusion in our simulation code was determined using the echo effect.
\end{abstract}

DOI: 10.1103/PhysRevSTAB.6.014205

PACS numbers: 29.27.Bd, 29.20.-c, 52.59.Sa, 52.65.Ff

\section{INTRODUCTION}

Spatial and temporal echo effects in plasmas have been known for a long time. Using the Vlasov-Poisson system, Gould, O'Neil, and Malmberg showed the possibility of generating collective plasma echoes by applying two external excitations to the plasma $[1,2]$. The existence of plasma echoes has been demonstrated experimentally by Malmberg et al. in Ref. [3]. The collisional damping of plasma echoes has been studied by Su and Oberman [4]. It was found that the collisional damping varies with time as $e^{-\nu \omega_{p}^{2} t^{3}}$, where $\nu$ and $\omega_{p}$ are the collisional and plasma frequencies, respectively. O'Neil showed that only the amplitude, but not the shape of the echo, depends on diffusion, and that when collisions are too frequent, the free-streaming perturbations will be smoothed out and the echo will be destroyed [5]. Jensen et al. measured the diffusion in velocity space of electrons due to microturbulance using plasma echoes [6]. For a review of echo measurements in plasmas, see Ref. [7].

In addition to collective plasma echoes that require a self-consistent treatment, there also exist ballistic plasma echoes. Ballistic echoes are essentially independent on the interaction between plasma particles and are due to free-streaming or ballistic particle motion only $[8,9]$.

Temporal ballistic echo phenomena are known to exist also in stored particle beams in both transverse and longitudinal planes of motion [10-12]. Longitudinal echo signals in coasting beams can be generated using two consecutive (delay time $\tau$ ) rf kicks at different harmonics $n_{1}$ and $n_{2}$. The interference of the two kicks leads to the echo signal at $t_{\text {echo }}=\tau n_{2} /\left(n_{2}-n_{1}\right)$ long after the signals associated with the two kicks have disappeared [13]. The occurrence of longitudinal echo phenomena in coasting proton beams has been observed in experiments in the Fermilab antiproton accumulator [14] and in the CERN SPS [15].

The diffusion rate in a stored antiproton beam was measured by Spentzouris, Ostiguy, and Colestock using longitudinal echoes [16]. The results of the echo measurements were successfully fitted to a formula that was obtained from a second-order perturbation analysis in the limit of ballistic motion, where impedance effects are negligible (see Ref. [13]). The effect of weak diffusion is found to modify the ballistic echo amplitude by an exponential factor that varies with the third power in time (see also Ref. [17]).

Here we present a theoretical study of collective beam echoes in the presence of the space-charge impedance and diffusion. Collective echoes in particle beams have not been studied before, even in the absence of diffusion. Experiments on echoes in ion beams including spacecharge effects will be possible, e.g., in electron cooler storage rings. In laboratory beams affected by space charge, collective phenomena, such as, e.g., turbulence, can cause diffusionlike effects, in addition to the diffusion rate generated by intrabeam scattering (see, e.g., [18]). The theoretical understanding of these phenomena in intense ion beams is still very preliminary. Therefore measurements of diffusion rates in such beams using echoes will be very helpful.

The paper is organized as follow: In Sec. II we start with a simplified analysis of the longitudinal echo mechanism in coasting beams. In Sec. III an expression for the time evolution of the beam echo amplitude will be derived using second-order perturbation theory. In Secs. III A and III B we derive closed form expressions for the ballistic and collective beam echoes, respectively. In Sec. IIIC we investigate the effect of weak diffusion on the ballistic and collective beam echo amplitudes. In Sec. IV we compare the analytic results with simulations. In Sec. V, we report the main conclusions.

\section{SIMPLIFIED MECHANISM FOR LONGITUDINAL BEAM ECHOES}

Let $\theta$ be the angular position of a given particle in a storage ring. When at time $t=0$ a voltage pulse of spatial 
dependence $\exp \left(\operatorname{in}_{1} \theta\right)$ is applied to a coasting beam, the resulting current modulation damps away. However, the distribution function is left with a perturbation of the form

$$
\delta f_{1}(\dot{\theta}, \theta, t)=f_{1}(\dot{\theta}) e^{i n_{1}(\theta-\dot{\theta} t)} .
$$

If after a time delay $\tau$ a second pulse at a different harmonic $n_{2}$ is applied to the beam the resulting current modulation will also damp away with its characteristic damping rate. At the same time it will modulate the unperturbed part of the distribution, leaving a first-order term of the form

$$
\delta f_{2}(\dot{\theta}, \theta, t)=f_{2}(\dot{\theta}) e^{i n_{2}[\theta-\dot{\theta}(t-\tau)]},
$$

and additionally modulating the perturbation due to the first pulse. The result is a second-order term of the form

$$
\delta f_{3}(\dot{\theta}, \theta, t)=f_{1}(\dot{\theta}) f_{2}(\dot{\theta}) e^{i\left[\left(n_{2}-n_{1}\right) \theta+n_{2} \dot{\theta} \tau-\left(n_{2}-n_{1}\right) \dot{\theta} t\right]} .
$$

Thus we see that at the time $t_{\text {echo }}=n_{2} \tau / \Delta n$ (with $\Delta n=$ $n_{2}-n_{1}$ ) the coefficient of $\dot{\theta}$ in the exponent vanishes and the velocity integral will not phase mix to zero. Hence a modulation at harmonic $\Delta n$ will appear on the beam. This echo signal appears long after the first two perturbations have damped away.

In order to estimate the effect of a velocity diffusion coefficient $D_{v}$ on the echo, where the velocity deviation from the synchronous particle is defined as $v=$ $R\left(\dot{\theta}-\omega_{0}\right)$ with the ring radius $R$ and the revolution frequency $\omega_{0}$, we use an effective collision frequency acting on the echo perturbation $\exp (i \Delta n \dot{\theta} t)$

$$
\tau_{\text {eff }}^{-1} \approx \frac{D_{v}}{R^{2}} \frac{\partial^{2}}{\partial \dot{\theta}^{2}} e^{i \Delta n \dot{\theta} t}=D_{v}\left(\frac{\Delta n t}{R}\right)^{2} .
$$

The effective damping of the echo signal is $\exp \left(-\tau_{\text {eff }}^{-1} t_{\text {echo }}\right)$. Echo formation requires

$$
\tau_{\text {eff }}^{-1} t_{\text {echo }}=D_{v}\left(\frac{\Delta n}{R}\right)^{2} t_{\text {echo }}^{3}<1 \Longleftrightarrow t_{\text {echo }}<\left(\frac{\tau_{f} R^{2}}{\Delta n v_{\text {rms }}^{2}}\right)^{1 / 3},
$$

where a diffusion rate is defined as $\tau_{f}^{-1}=D_{v} / v_{\mathrm{rms}}^{2}$, with the rms velocity spread $v_{\text {rms }}$ of the beam particles. Otherwise the echo effect will be destroyed by diffusion.

\section{SELF-CONSISTENT SECOND-ORDER PERTURBATION THEORY}

For our detailed investigation of beam echoes, we start from the Vlasov equation for the longitudinal distribution function $f(\epsilon, \theta, t)$ with an additional diffusion term

$$
\frac{\partial f(\epsilon, \theta, t)}{\partial t}+\dot{\theta} \frac{\partial f(\epsilon, \theta, t)}{\partial \theta}+\dot{\epsilon} \frac{\partial f(\epsilon, \theta, t)}{\partial \epsilon}=\frac{D_{v}}{R^{2}} \frac{\partial^{2} f}{\partial \dot{\theta}^{2}} .
$$

Here $\epsilon=E-E_{0}$ is the energy deviation from the synchronous particle energy. The relationship between $\epsilon$ and the velocity deviation $v$ is given through $v=$ $-\eta \epsilon /\left(R \beta_{0} c \gamma_{0} M\right)$, with the relativistic factors $\beta_{0}$ and $\gamma_{0}$, the frequency slip factor $\eta$, and the particle rest mass $M$.

In the presence of an externally applied voltage $V^{\operatorname{ext}}(\theta, t)$ and of the longitudinal space-charge impedance $Z_{\|}^{n}=Z_{\|}\left(n \omega_{0}\right)$ (see, e.g., [19]), the time derivative of the energy deviation $\dot{\boldsymbol{\epsilon}}(\theta, t)$ is (see, e.g., Refs. [20,21])

$$
\begin{aligned}
\dot{\boldsymbol{\epsilon}}(\theta, t)=-\frac{q \omega_{0}}{2 \pi}[ & V^{\mathrm{ext}}(\theta, t) \\
& \left.+\frac{q \omega_{0}}{2 \pi} \sum Z_{\|}^{n} e^{i n \theta} \int_{-\infty}^{\infty} f_{n}(\epsilon, t) d \epsilon\right],
\end{aligned}
$$

where $q$ is the single particle charge, $n$ is the harmonic number, and $f_{n}$ is the Fourier amplitude of the distribution function.

Let the distribution function $f(\epsilon, \theta, t)$ be composed of three parts: the unperturbed equilibrium part $f_{0}(\epsilon)$, a first-order perturbation $f^{(1)}(\epsilon, \theta, t)$, and $f^{(2)}(\epsilon, \theta, t)$ a second-order perturbation. In accordance we introduce a first-order $\dot{\boldsymbol{\epsilon}}$

$$
\dot{\boldsymbol{\epsilon}}^{(1)}(\theta, t) \approx-\frac{q \omega_{0}}{2 \pi}\left[V^{\mathrm{ext}}(\theta, t)+\frac{q \omega_{0}}{2 \pi} \sum_{n=-\infty}^{\infty} Z_{\|}^{n} e^{i n \theta} \int_{-\infty}^{\infty} f_{n}^{(1)}(\epsilon, t) d \epsilon\right] \equiv \dot{\boldsymbol{\epsilon}}^{(0)}(\theta, t)-\left(\frac{q \omega_{0}}{2 \pi}\right)^{2} \sum_{n=-\infty}^{\infty} Z_{\|}^{n} e^{i n \theta} \int_{-\infty}^{\infty} f_{n}^{(1)}(\epsilon, t) d \epsilon
$$

In the following analysis, Eqs. (6) and (8) will be solved by iteration for the case $D_{v}=0$ using the complex Fourier representation in the azimuthal variable $\theta$ and the Laplace transform in time. Therefore Eqs. (6) and (8) are multiplied by $e^{-s t-i n \theta}$, where $s$ is the Laplace transform parameter. Integrating over $\theta$ and $t$ gives

$f_{n}(\epsilon, s)=-\frac{1}{s+i n \dot{\theta}} \frac{\partial}{\partial \epsilon} \int_{0}^{\infty} \int_{0}^{2 \pi} \frac{d t d \theta}{2 \pi} e^{-s t-i n \theta} f(\epsilon, \theta, t) \dot{\boldsymbol{\epsilon}}^{(1)}(\theta, t)=-\frac{1}{s+i n \dot{\theta}} \frac{\partial}{\partial \epsilon} \int_{\sigma-i \infty}^{\sigma+i \infty} d s_{1} \sum_{\ell=-\infty}^{\infty} \dot{\boldsymbol{\epsilon}}_{\ell}^{(1)}(s) f_{n-\ell}\left(\epsilon, s-s_{1}\right)$,

where the Fourier-Laplace transform of any function $G(\epsilon, \theta, t)$ and its inverse are defined as follows:

$$
G_{n}(\epsilon, s)=\int_{0}^{\infty} \int_{0}^{2 \pi} \frac{d t d \theta}{2 \pi} e^{-s t-i n \theta} G(\epsilon, \theta, t)
$$




$$
G(\epsilon, \theta, t)=\int_{\sigma-i \infty}^{\sigma+i \infty} \frac{d s}{2 \pi i} e^{s t} \sum_{n=-\infty}^{\infty} e^{i n \theta} G_{n}(\epsilon, s)
$$

Here $\sigma$ is a vertical line in the complex $s$ plane with $\operatorname{Re} \sigma>0$ and to the right of all possible singularities. Substituting the unperturbed distribution $f_{0}(\epsilon)$ on the right-hand side of Eq. (9) gives for the first-order distribution function

$$
f_{n}^{(1)}(\epsilon, s)=-\frac{1}{s+i n \dot{\theta}} \frac{\dot{\epsilon}_{n}^{(0)}(s)}{\varepsilon[s, n]} \frac{\partial f_{0}(\epsilon)}{\partial \epsilon}=-\frac{1}{s+i n \dot{\theta}} \frac{1}{\varepsilon[s, n]} \frac{\partial}{\partial \epsilon} \int_{0}^{\infty} \int_{0}^{2 \pi} \frac{d t d \theta}{2 \pi} e^{-s t-i n \theta} \dot{\boldsymbol{\epsilon}}^{(0)}(\theta, t) f_{0}(\epsilon),
$$

and

$$
\begin{gathered}
\dot{\epsilon}_{n}^{(1)}(s)=\frac{\dot{\epsilon}_{n}^{(0)}(s)}{\varepsilon[s, n]} \\
\varepsilon[s, n]=1-Z_{\|}^{n}\left(\frac{q \omega_{0}}{2 \pi}\right)^{2} \int_{-\infty}^{\infty} \frac{d \epsilon}{s+i n \dot{\theta}} \frac{\partial f_{0}(\epsilon)}{\partial \epsilon} .
\end{gathered}
$$

Making use of the linear solutions $f_{n}^{(1)}(\epsilon, s)$ and $\dot{\boldsymbol{\epsilon}}_{n}^{(1)}(s)$ in Eqs. (12) and (13), we obtain the following expression for the second-order distribution function $f_{n}^{(2)}(\epsilon, s)$ :

$$
\begin{aligned}
f_{n}^{(2)}(\epsilon, s) & =-\frac{1}{s+i n \dot{\theta}} \frac{1}{\varepsilon[s, n]} \frac{\partial}{\partial \epsilon} \int_{0}^{\infty} \int_{0}^{2 \pi} \frac{d t d \theta}{2 \pi} e^{-s t-i n \theta} \dot{\boldsymbol{\epsilon}}^{(1)}(\theta, t) f^{(1)}(\epsilon, \theta, t) \\
& =-\frac{1}{2 \pi \varepsilon(s, n)} \frac{1}{s+i n \dot{\theta}} \frac{\partial}{\partial \boldsymbol{\epsilon}} \int_{\sigma-i \infty}^{\sigma+i \infty} d s_{1} \sum_{\ell=-\infty}^{\infty} \dot{\boldsymbol{\epsilon}}_{\ell}^{(1)}(s) f_{n-\ell}^{(1)}\left(\epsilon, s-s_{1}\right) \\
& =\frac{q \omega_{0}}{4 \pi^{2} \varepsilon(s, n)} \frac{1}{s+i n \dot{\theta}} \frac{\partial}{\partial \boldsymbol{\epsilon}} \int_{\sigma-i \infty}^{\sigma+i \infty} d s_{1} \sum_{\ell=-\infty}^{\infty} \frac{\dot{\boldsymbol{\epsilon}}_{\ell}^{(1)}(s) V_{n-\ell}^{\mathrm{ext}}\left(s-s_{1}\right)}{s-s_{1}+i(n-\ell) \dot{\theta}} \frac{1}{\varepsilon\left(s-s_{1}, n-\ell\right)} \frac{\partial f_{0}}{\partial \boldsymbol{\epsilon}} \\
& =-\frac{q^{2} \omega_{0}^{2}}{8 \pi^{3} \varepsilon(s, n)} \frac{1}{s+i n \dot{\theta}} \frac{\partial}{\partial \epsilon} \int_{\sigma-i \infty}^{\sigma+i \infty} d s_{1} \sum_{\ell=-\infty}^{\infty} \frac{V_{\ell}^{\text {ext }}(s) V_{n-\ell}^{\text {ext }}\left(s-s_{1}\right)}{s-s_{1}+i(n-\ell) \dot{\theta}} \frac{1}{\varepsilon(s, \ell) \varepsilon\left(s-s_{1}, n-\ell\right)} \frac{\partial f_{0}}{\partial \boldsymbol{\epsilon}} .
\end{aligned}
$$

The second-order current perturbation $I_{n}^{(2)}(s)$ can be calculated from Eq. (15) as follows:

$$
\begin{aligned}
I_{n}^{(2)}(s) & =\frac{q \omega_{0}}{2 \pi} \int_{-\infty}^{\infty} d \epsilon f_{n}^{(2)}(\epsilon, s) \\
& =-\frac{q^{3} \omega_{0}^{3}}{16 \pi^{4}} \int_{-\infty}^{\infty} \frac{d \epsilon}{s+i n \dot{\theta}} \frac{1}{\varepsilon[s, n]} \frac{\partial}{\partial \epsilon} \int_{\sigma-i \infty}^{\sigma+i \infty} d s_{1} \sum_{\ell=-\infty}^{\infty} \frac{V_{\ell}^{\mathrm{ext}}\left(s_{1}\right) V_{n-\ell}^{\mathrm{ext}}\left(s-s_{1}\right)}{s-s_{1}+i(n-\ell) \dot{\theta}} \frac{1}{\varepsilon\left[s_{1}, \ell\right] \varepsilon\left[s-s_{1}, n-\ell\right]} \frac{\partial f_{0}}{\partial \epsilon} .
\end{aligned}
$$

Changing the variable $\epsilon$ into $\dot{\theta}$ such that [22]

$$
\frac{\partial f_{0}(\epsilon)}{\partial \epsilon} d \epsilon=\frac{\partial f_{0}(\dot{\theta})}{\partial \dot{\theta}} \frac{d \dot{\theta}}{d \epsilon} d \dot{\theta}, \quad \frac{d \dot{\theta}}{d \epsilon}=-\frac{\eta}{\beta_{0}^{2}} \frac{\omega_{0}}{\gamma_{0} M c^{2}},
$$

and introducing the half-width of the angular distribution function $f_{0}(\dot{\theta})$ measured at half-height $S=\Delta \dot{\theta} / 2$ such that

$$
\dot{\theta}-\omega_{0}=x S, \quad \int_{-\infty}^{\infty} f_{0}(x) d x=1,
$$

where $x$ is a dimensionless variable, Eq. (16) becomes

$$
\begin{aligned}
I_{n}^{(2)}(s)= & -\frac{q^{3} \omega_{0}^{3} N_{0}}{16 \pi^{4}}\left(\frac{\eta}{\beta_{0}^{2}} \frac{\omega_{0}}{\gamma_{0} M c^{2} S}\right)^{2} \int_{-\infty}^{\infty} \frac{d x}{s+i n \omega_{0}+i n S x} \frac{1}{\varepsilon[s, n]} \frac{\partial}{\partial x} \\
& \times\left[\frac{\partial f_{0}(x)}{\partial x} \int_{\sigma-i \infty}^{\sigma+i \infty} d s_{1} \sum_{\ell=-\infty}^{\infty} \frac{V_{\ell}^{\operatorname{ext}}\left(s_{1}\right) V_{n-\ell}^{\mathrm{ext}}\left(s-s_{1}\right)}{s-s_{1}+i(n-\ell)\left(\omega_{0}+S x\right)} \frac{1}{\varepsilon\left[s_{1}, \ell\right] \varepsilon\left[s-s_{1}, n-\ell\right]}\right],
\end{aligned}
$$


where $N_{0}$ is the number of particles in the beam. The relation between the half-width of the angular distribution function $S$ and its full momentum spread $\Delta P$ at half-height is given by $2 S=-\eta \omega_{0} \sigma_{p}^{\mathrm{FWHM}}$, where $\sigma_{p}^{\mathrm{FWHM}}=\left(\Delta P / P_{0}\right)_{\mathrm{FWHM}}$ is the relative momentum spread at full width at half maximum.

In the following analysis we assume two external excitations of the form

$$
V^{\operatorname{ext}}(\theta, t)=V_{1} \cos n_{1} \theta \delta\left[\frac{\omega_{0}}{2 \pi} t\right]+V_{2} \cos n_{2} \theta \delta\left[\frac{\omega_{0}}{2 \pi}(t-\tau)\right]
$$

where $\left(V_{1}, V_{2}\right)$ and $\left(n_{1}, n_{2}\right)$ are the amplitudes and harmonic numbers of the rf kicks, respectively. The excitations are switched on at time $t=0$ and at a delay time $t=\tau$. Using the following Laplace-Fourier representation of $V^{\text {ext }}(\theta, t)$ :

$$
V_{\ell}^{\mathrm{ext}}(s)=\frac{V_{1} \pi}{\omega_{0}}\left[\delta_{\ell,-n_{1}}+\delta_{\ell, n_{1}}\right]+\frac{V_{2} \pi}{\omega_{0}}\left[\delta_{\ell,-n_{2}}+\delta_{\ell, n_{2}}\right] e^{-s \tau},
$$

the product term $V_{\ell}^{\text {ext }}\left(s_{1}\right) V_{n-\ell}^{\text {ext }}\left(s-s_{1}\right)$ becomes

$$
\begin{aligned}
V_{\ell}^{\mathrm{ext}}\left(s_{1}\right) V_{n-\ell}^{\mathrm{ext}}\left(s-s_{1}\right)=\frac{V_{1} V_{2} \pi^{2}}{\omega_{0}^{2}}[ & \left(\delta_{\ell,-n_{2}} \delta_{n-\ell,-n_{1}}+\delta_{\ell,-n_{2}} \delta_{n-\ell, n_{1}}+\delta_{\ell, n_{2}} \delta_{n-\ell,-n_{1}}+\delta_{\ell, n_{2}} \delta_{n-\ell, n_{1}}\right) e^{-s_{1} \tau} \\
& \left.+\left(\delta_{\ell,-n_{1}} \delta_{n-\ell,-n_{2}}+\delta_{\ell,-n_{1}} \delta_{n-\ell, n_{2}}+\delta_{\ell, n_{1}} \delta_{n-\ell,-n_{2}}+\delta_{\ell, n_{1}} \delta_{n-\ell, n_{2}}\right) e^{-\left(s-s_{1}\right) \tau}\right]
\end{aligned}
$$

In calculating $V_{\ell}^{\text {ext }}\left(s_{1}\right) V_{n-\ell}^{\text {ext }}\left(s-s_{1}\right)$, only mixed terms that are proportional to $V_{1} V_{2}$ have been considered. Upon substituting for $V_{\ell}^{\text {ext }}\left(s_{1}\right) V_{n-\ell}^{\text {ext }}\left(s-s_{1}\right)$ from Eq. (22) into Eq. (19) and back transforming $I_{n}^{(2)}(s)$ into $I^{(2)}(\theta, t)$ such that

$$
I^{(2)}(\theta, t)=\frac{1}{2 \pi i} \int_{\sigma-i \infty}^{\sigma+i \infty} d s e^{s t} \sum_{n=-\infty}^{\infty} e^{i n \theta} I_{n}^{(2)}(s),
$$

we obtain the following expression for the second-order beam echo:

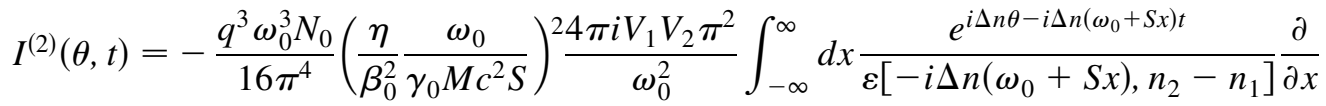

$$
\begin{aligned}
& \times\left[\frac{\partial f_{0}(x)}{\partial x} \frac{e^{i n_{2}\left(\omega_{0}+S x\right) \tau}}{\varepsilon\left[i n_{1}\left(\omega_{0}+S x\right),-n_{1}\right] \varepsilon\left[-i n_{2}\left(\omega_{0}+S x\right), n_{2}\right]}\right]+\text { c.c., }
\end{aligned}
$$

where c.c. denotes the complex conjugate. In arriving at Eq. (23), only terms that contain $\pm\left(n_{2}-n_{1}\right)$ have been considered, and therefore, only terms of the harmonic difference $\pm \Delta n= \pm\left(n_{2}-n_{1}\right)$ can contribute to the echo problem. In Secs. III A and III B, Eq. (23) will be used to obtain the ballistic and collective echo envelope functions in the absence of diffusion effects, respectively.

\section{A. Ballistic beam echoes}

In the absence of space-charge effects, $Z_{\|}^{n}=0$, the longitudinal dielectric function in Eq. (14) becomes $\varepsilon=1$. Accordingly, Eq. (23) becomes

$$
I^{(2)}(\theta, t)=-\frac{q^{3} \omega_{0}^{3} N_{0}}{16 \pi^{4}}\left(\frac{\eta}{\beta_{0}^{2}} \frac{\omega_{0}}{\gamma_{0} M c^{2} S}\right)^{24 \pi i V_{1} V_{2} \pi^{2}} \int_{-\infty}^{\infty} d x e^{i \Delta n \theta-i \Delta n\left(\omega_{0}+S x\right) t} \frac{\partial}{\partial x}\left[\frac{\partial f_{0}(x)}{\partial x} e^{i n_{2}\left(\omega_{0}+S x\right) \tau}\right]+\text { c.c. }
$$

To obtain an expression for the second-order perturbation current as a function of time only, we introduce the following complex Fourier series representation of $I^{(2)}(\theta, t)$ in terms of the harmonic difference $\Delta n=n_{2}-n_{1}$ :

$$
I^{(2)}(\theta, t)=\sum_{\Delta n=-\infty}^{\infty} I_{\Delta n}^{(2)}(t) e^{i \Delta n \theta}, \quad I_{\Delta n}^{(2)}(t)=\frac{1}{2 \pi} \int_{0}^{2 \pi} I^{(2)}(\theta, t) e^{-i \Delta n \theta} d \theta,
$$

where $I_{\Delta n}^{(2)}(t)$ is the echo amplitude at harmonic difference $\Delta n=n_{2}-n_{1}$. Assuming a Gaussian equilibrium distribution $f_{0}(x)$ such that

$$
f_{0}(x)=\frac{R S}{\sqrt{2 \pi} \delta v} e^{-\left[\left(R^{2} S^{2}\right) /\left(2 \delta v^{2}\right)\right] x^{2}}, \quad \delta v=\eta \beta_{0} c \sigma_{p}^{\mathrm{rms}},
$$

where $\sigma_{p}^{\mathrm{rms}}=\sigma_{p}=\left(\Delta P / P_{0}\right)_{\mathrm{rms}}$ is the rms relative momentum spread, and upon integrating over $x$, the time evolution of the echo becomes 


$$
I_{\Delta n}^{(2)}(t, \theta)=I_{\Delta n}^{(2)}(t) \sin \left[\omega_{0} \Delta n\left(t-t_{\text {echo }}\right)-\Delta n \theta\right]
$$

with the echo amplitude

$$
I_{\Delta n}^{(2)}(t)=\frac{V_{1} V_{2} q^{3} \omega_{0}^{3} N_{0}}{2 \pi \omega_{0}^{2}}\left(\frac{\eta}{\beta_{0}^{2}} \frac{\omega_{0}}{\gamma_{0} M c^{2}}\right)^{2} \Delta n t\left(n_{2} \tau-\Delta n t\right) e^{-\left\{\left[\eta^{2} \beta_{0}^{2} c^{2} \sigma_{p}^{2}\left(n_{2} \tau-\Delta n t\right)^{2}\right] /\left(2 R^{2}\right)\right\}} .
$$

Equation (28) shows that the echo amplitude is varying as $q^{3} \omega_{0}^{3}$ in the absence of space-charge effects $[1,16] . \quad$ For $\quad E_{0}=\gamma M c^{2}, \quad t_{\text {echo }}=\left[\left(n_{2} \tau\right) /(\Delta n)\right], \quad I_{0}=$ $\left[\left(N_{0} q \beta c\right) /(2 \pi R)\right], \omega_{0}=(\beta c / R), \chi=\left\{\left[\eta \beta c \sigma_{p} \Delta n \times(t-\right.\right.$ $\left.\left.\left.t_{\text {echo }}\right)\right] / R\right\}$, and $(\Delta \sigma)^{2}=\left[\left(q^{2} V_{1} V_{2}\right) /\left(\beta^{4} E_{0}^{2}\right)\right]$, Eq. (28) becomes

$$
I_{\Delta n}^{(2)}(t)=\frac{I_{0}}{2}(\Delta \sigma)^{2} \frac{\eta \beta c \Delta n t}{R \sigma_{p}} \chi e^{-\left(\chi^{2} / 2\right)}
$$

The echo amplitude Eq. (29) has a double peak structure with unequal heights (see Fig. 1). The unequal heights of the ballistic echo peaks have already been reported in the literature $[13,23]$. This behavior is due to the appearance of the factor $\Delta n t$ in Eq. (29). By finding the maxima of the echo amplitude Eq. (29) with respect to time we obtain the two echo times $t_{+}$and $t_{-}$

$t_{ \pm}=\frac{2}{3} \frac{n_{2}}{n_{2}-n_{1}} \tau-2 a \cos \left(\frac{2 \pi \pm \phi}{3}\right), \quad \phi=\cos ^{-1} \frac{b}{a^{3}}$,

$$
\begin{aligned}
& a=\frac{\operatorname{sgn}(b)}{3} \sqrt{n_{2}^{2} \tau^{2}+\frac{6 R^{2}}{\eta^{2} \beta_{0}^{2} c^{2} \sigma_{p}^{2}},} \\
& b=n_{2} \tau\left(\frac{R^{2}}{6 \eta^{2} \beta_{0}^{2} c^{2} \sigma_{p}^{2}}-\frac{n_{2}^{2} \tau^{2}}{27}\right) .
\end{aligned}
$$

The time spacing between the left and the right echo peaks $\Delta t_{\text {peak }}=t_{+}-t_{-}$can be obtained analytically from Eq. (30), namely,

$$
\Delta t_{\text {peak }}=t_{+}-t_{-}=2 \sqrt{3} a \sin \frac{\phi}{3} .
$$

Equation (32) gives the time spacing between the left and the right echo peaks. The time spacing $\Delta t_{\text {peak }}$ is determined by a nonlinear function of the delay time $\tau$. It can be seen from Eq. (32) that the peak spacings are inversely proportional to the initial relative rms momen- tum spread $\sigma_{p}$ or the beam energy spread, as pointed out by Spentzouris et al. [16]. The maximum echo amplitude is then determined through

$$
I_{\Delta n}^{\max }=I_{\Delta n}^{(2)}\left(t=t_{+}\right)
$$

\section{B. Collective echoes}

In this section we calculate the current $I^{(2)}(\theta, t)$ in Eq. (23) for the case $Z_{\|}^{n} \neq 0$. Following Gould et al. [1], and for a Gaussian equilibrium distribution function in Eq. (26) the current $I^{(2)}(\theta, t)$ is obtained for $t<$ $\left[\left(n_{2}\right) /\left(n_{2}-n_{1}\right)\right] \tau$ by closing the contour in the upper half plane and by picking up poles from the Landau roots of $\varepsilon\left[i n_{1}\left(\omega_{0}+S x\right),-n_{1}\right]$, where $\varepsilon[s, n]$ denotes the longitudinal dielectric function defined in Eq. (14). For $t>$ $\left[\left(n_{2}\right) /\left(n_{2}-n_{1}\right)\right] \tau$, we close the contour in the lower half plane and pick up poles from the Landau roots of $\varepsilon\left[-i n_{2}\left(\omega_{0}+S x\right), n_{2}\right]$ and $\varepsilon\left[-i \Delta n\left(\omega_{0}+S x\right), \Delta n\right]$.

Introducing the abbreviations $A_{0}$ and $\gamma_{L}(n)$ such that

$$
\begin{gathered}
A_{0}=\sqrt{2 \pi} \frac{I_{0}}{4} \alpha\left(\Delta \sigma_{p}\right)^{2}\left(\frac{\eta \beta c}{\delta v}\right)^{2}, \\
\gamma_{L}(n)=-\left.\frac{\operatorname{Im}(\varepsilon)}{\partial \varepsilon / \partial \Omega}\right|_{\Omega=\Omega_{\text {real }}}=-\sqrt{\frac{\pi}{8}} \alpha^{3} n S U_{n}^{2} e^{-\left(\alpha^{2} / 2\right) U_{n},} \\
U_{n}=-\frac{2 I_{0} q}{\pi \gamma_{0} \beta_{0}^{2} M c^{2} \eta\left(\sigma_{p}^{\mathrm{FWHM}}\right)^{2}} \operatorname{Im} \frac{Z_{\|}^{n}}{n}
\end{gathered}
$$

where $U_{n}$ is the normalized longitudinal space-charge impedance [22] and $\gamma_{L}(n)$ is the Landau damping coefficient, the second-order current associated with the echo pulse in the presence of space-charge effects becomes

$$
I_{\Delta n}^{(2)}(\theta, t)=A_{0}\left\{\begin{array}{ccc}
2 c_{n_{1}} e^{-\left[\left(\alpha^{2} U_{n 1}\right) / 2\right]} e^{\left\{\left[\gamma_{L}\left(n_{1}\right) \Delta n\right] /\left(n_{1}\right)\right\}\left[\left(n_{2} / \Delta n\right) \tau-t\right]} \sin \left[\omega(\Delta n)\left(\frac{n_{2}}{\Delta n} \tau-t\right)-\Delta n \theta+\gamma_{n_{1}}\right], & t<\frac{n_{2} \tau}{\Delta n}, \\
c_{\Delta n} e^{-\left[\left(\alpha^{2} U_{\Delta n}\right) / 2\right]} e^{\gamma_{L}(\Delta n)\left[t-\left(n_{2} / \Delta n\right) \tau\right]} \sin \left[\omega(\Delta n)\left(t-\frac{n_{2} \tau}{\Delta n}\right)-\Delta n \theta+\gamma_{\Delta n}\right] & \\
& +c_{n_{2}} e^{-\left[\left(\alpha^{2} U_{n 2}\right) / 2\right]} e^{\left\{\left[\gamma_{L}\left(n_{2}\right) \Delta n\right] /\left(n_{2}\right)\right\}\left[t-\left(n_{2} \tau\right) /(\Delta n)\right]} \sin \left[\omega(\Delta n)\left(t-\frac{n_{2} \tau}{\Delta n}\right)-\Delta n \theta+\gamma_{n_{2}}\right], & t>\frac{n_{2} \tau}{\Delta n},
\end{array}\right.
$$

with the frequency of space-charge waves $\omega(n) \approx n \omega_{0} \pm n S \sqrt{U_{n}}$. The different parameters in Eq. (37) are defined as follows:

$$
\Delta n=n_{2}-n_{1}, \quad \alpha=\frac{S R}{\delta v}, \quad S=-0.5 \eta \omega_{0}\left(\sigma_{p}\right)_{\mathrm{FWHM}}, \quad \delta v=|\eta| \beta_{0} c\left(\sigma_{p}\right)_{\mathrm{rms}},
$$




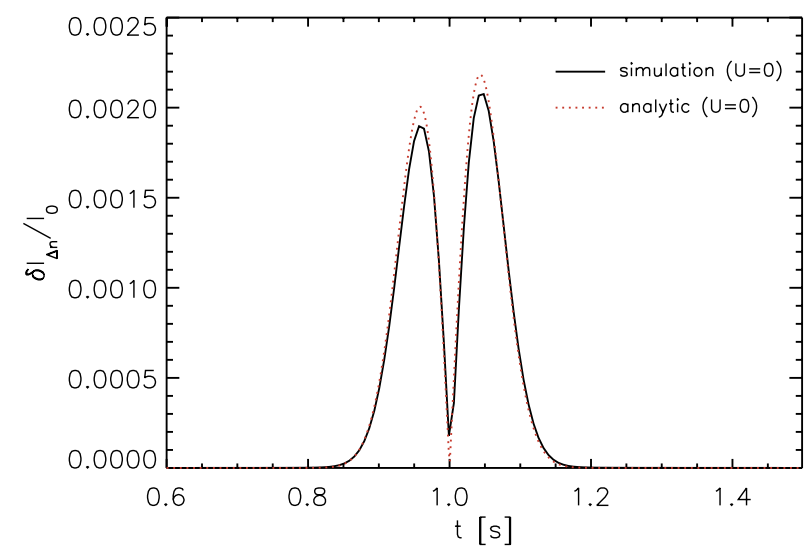

FIG. 1. (Color) Comparison of the simulation result (solid line) with the analytic result (red dotted line) for the ballistic echo amplitude $(U=0)$ versus time. The delay time between the two rf kicks is $\tau=0.1 \mathrm{~s}$.

$$
\begin{gathered}
\tan \gamma_{\Delta n}=\frac{a_{\Delta n}}{b_{\Delta n}}, \quad \tan \gamma_{n_{2}}=\frac{a_{n_{2}}}{b_{n_{2}}}, \quad \tan \gamma_{n_{1}}=\frac{a_{n_{1}}}{b_{n_{1}}}, \\
c_{\Delta n}=\sqrt{a_{\Delta n}^{2}+b_{\Delta n}^{2}}, \quad c_{n_{2}}=\sqrt{a_{n_{2}}^{2}+b_{n_{2}}^{2}}, \\
c_{n_{1}}=\sqrt{a_{n_{1}}^{2}+b_{n_{1}}^{2}}, \\
a_{\Delta n}=\sqrt{U_{\Delta n}}\left(1-\alpha^{2} U_{\Delta n}\right), \quad b_{\Delta n}=n_{2} S \tau U_{\Delta n}, \\
a_{n 1}=\sqrt{U_{n 1}}\left(1-\alpha^{2} U_{n 1}\right), \quad b_{n 1}=n_{2} S \tau U_{n 1}, \\
a_{n 2}=\sqrt{U_{n 2}}\left(1-\alpha^{2} U_{n 2}\right), \quad b_{n 2}=n_{2} S \tau U_{n 2} .
\end{gathered}
$$

From Eq. (38) we notice that the collective echo amplitude will grow and decay exponentially by a rate determined by the Landau damping rates $\gamma_{L}(\Delta n)$, $\left\{\left[\gamma_{L}\left(n_{2}\right) \Delta n\right] /\left(n_{2}\right)\right\}$, and $\left\{\left[\gamma_{L}\left(n_{1}\right) \Delta n\right] /\left(n_{1}\right)\right\}$. This asymmetric behavior of the collective echo envelope function is well known from the studies of the plasma wave echoes and was first obtained by Gould et al. [1]. An important distinction between electron plasma echoes and beam echoes is that in beams the transition between the ballistic and collective echoes regime is controlled by the intensity parameter $U_{n}$, whereas in electron plasmas the relevant parameter is the wavelength (see, e.g., [9]). It is important to point out that in the analytic approach of calculating the collective beam echo response, the Landau damping times for all three modes have been assumed to be short relative to the delay time $\tau$ between the rf excitations (see Ref. [1]). Therefore, the adopted analytical approach in calculating the collective beam echo might fail for large space-charge impedances $U_{n}$.

In the case of constant space-charge parameter $U=$ $U_{n_{1}}=U_{n_{2}}=U_{\Delta n}$, Eq. (37) simplifies considerably and the echo can be written as

$$
I_{\Delta n}^{(2)}(\theta, t)=I_{\Delta n}^{(2)}(t) \sin \left[\omega(n)\left(t-t_{\text {echo }}\right)-\Delta n \theta+\gamma\right]
$$

with the echo amplitude

$$
I_{\Delta n}^{(2)}(t)=A_{1} \begin{cases}e^{-\left[\left(\alpha^{2} U\right) / 2\right]} e^{\gamma_{L}(\Delta n)\left(t_{\text {echo }}-t\right)} & t<t_{\text {echo }}, \\ e^{-\left[\left(\alpha^{2} U_{\Delta n}\right) / 2\right]} e^{\gamma_{L}(\Delta n)\left(t-t_{\text {echo }}\right)} & t>t_{\text {echo }}\end{cases}
$$

and

$$
A_{1}=2 A_{0}\left[U\left(1+\alpha^{2} U\right)^{2}+\left(n_{2} S \tau U\right)^{2}\right]^{1 / 2} .
$$

The maximum echo amplitude at $t=t_{\text {echo }}$ is given through

$$
I_{\Delta n}^{\max }=A_{1} e^{-\left[\left(\alpha^{2} U\right) /(2)\right]}
$$

\section{Longitudinal beam echoes with diffusion}

In this section we investigate the effect of diffusion on longitudinal ballistic and collective beam echoes. According to our second-order iteration procedure for the Vlasov equation, the second-order distribution function, including diffusion effects, will be derived from the following equation:

$$
\left[\frac{\partial}{\partial t}+\dot{\theta} \frac{\partial}{\partial \theta}-\frac{D_{v}}{R^{2}} \frac{\partial^{2}}{\partial \dot{\theta}^{2}}\right] f^{(2)}(\dot{\theta}, \theta, t)=-\dot{\boldsymbol{\epsilon}}^{(1)}(\theta, t) \frac{\partial f^{(1)}(\epsilon, \theta, t)}{\partial \epsilon} .
$$

The right-hand side of Eq. (48) is the source term for the time rate of change of the second-order distribution function $f^{(2)}(\dot{\theta}, \theta, t)$. By ignoring the effect of diffusion on the first-order perturbed distribution function $f^{(1)}(\dot{\theta}, \theta, t)$ in Eq. (48), the right-hand side of Eq. (48) becomes the time derivative of the second-order distribution function for $D_{v}=0$

$$
\frac{d F^{(2)}}{d t}=-\dot{\boldsymbol{\epsilon}}^{(1)}(\theta, t) \frac{\partial f^{(1)}(\epsilon, \theta, t)}{\partial \epsilon} .
$$

Here $F^{(2)}$ denotes the second-order distribution function for $D_{v}=0$ given in Eq. (15). Transforming $f^{(2)}(\dot{\theta}, \theta, t)$ in Eq. (48) such that

$$
\begin{aligned}
f^{(2)}(\dot{\theta}, \theta, t) & =\sum_{n} e^{i n \theta} f_{n}^{(2)}(\dot{\theta}, t) \\
& =\sum_{n} e^{i n \theta+i n \dot{\theta} t} h_{n}(\dot{\theta}, t),
\end{aligned}
$$

where $h_{n}(\dot{\theta}, t)$ is a slowly varying function with $\dot{\theta}$ such that $\partial^{2} h / \partial \dot{\theta}^{2} \approx 0$, and upon solving for $h_{n}(\dot{\theta}, t)$ results in the following expression for $f_{n}^{(2)}(\dot{\theta}, t)$ : 


$$
f_{n}^{(2)}(\dot{\theta}, t)=e^{i n \dot{\theta} t} h_{n}(\dot{\theta}, t)=-e^{-2 i n \dot{\theta} t-\left[\left(D_{v} n^{2} t^{3}\right) /\left(3 R^{2}\right)\right]} \int_{-\infty}^{t} d t^{\prime \prime} \frac{d F_{n}^{(2)}\left(\dot{\theta}, t^{\prime \prime}\right)}{d t^{\prime \prime}} e^{2 i n \dot{\theta} t^{\prime \prime}+\left[\left(D_{v} n^{2} t^{\prime \prime 3}\right) /\left(3 R^{2}\right)\right]}
$$

In order to evaluate Eq. (51) we first rewrite Eq. (15) for $F_{n}^{(2)}$ by transforming the variables from $\epsilon$ into $\dot{\theta}$ and then from $\dot{\theta}$ into $x$

$$
\begin{aligned}
F_{n}^{(2)}(x, s)= & -\frac{q^{2} \omega_{0}^{2}}{8 \pi^{3}}\left(\frac{\eta}{\beta_{0}^{2}} \frac{\omega_{0}}{\gamma_{0} M c^{2} S}\right)^{2} \frac{N_{0}}{s+i n \omega_{0}+i n S x} \frac{1}{\varepsilon[s, n]} \frac{\partial}{\partial x} \\
& \times\left[\frac{\partial f_{0}(x)}{\partial x} \int_{\sigma-i \infty}^{\sigma+i \infty} d s_{1} \sum_{\ell=-\infty}^{\infty} \frac{V_{\ell}^{\text {ext }}\left(s_{1}\right) V_{n-\ell}^{\text {ext }}\left(s-s_{1}\right)}{s-s_{1}+i(n-\ell)\left(\omega_{0}+S x\right)} \frac{1}{\varepsilon\left[s_{1}, \ell\right] \varepsilon\left[s-s_{1}, n-\ell\right]}\right] .
\end{aligned}
$$

Upon substituting for $V_{\ell}^{\text {ext }}\left(s_{1}\right) V_{n-\ell}^{\text {ext }}\left(s-s_{1}\right)$ from Eq. (22), summing over $\ell$, integrating over $s_{1}$, and Laplace back transforming from $s$ into $t$, selecting terms such that $n= \pm \Delta n= \pm\left(n_{2}-n_{1}\right)$, Eq. (52) becomes

$$
\begin{aligned}
F_{n}^{(2)}(x, t)=- & 2 \pi i \frac{q^{2} \omega_{0}^{2} N_{0}}{8 \pi^{3}}\left(\frac{\eta}{\beta_{0}^{2}} \frac{\omega_{0}}{\gamma_{0} M c^{2} S}\right)^{2} \frac{V_{1} V_{2} \pi^{2}}{\omega_{0}^{2}} \frac{e^{-i n\left(\omega_{0}+S x\right) t}}{\varepsilon\left[-i n\left(\omega_{0}+S x\right), n\right]} \\
\times & \frac{\partial}{\partial x} \frac{\partial f_{0}(x)}{\partial x} \frac{\delta_{n, n_{1}-n_{2}} e^{-i n_{2}\left(\omega_{0}+S x\right) \tau}}{\varepsilon\left[i n_{2}\left(\omega_{0}+S x\right),-n_{2}\right] \varepsilon\left[-i\left(n+n_{2}\right)\left(\omega_{0}+S x\right), n+n_{2}\right]} \\
& +\frac{\partial}{\partial x} \frac{\partial f_{0}(x)}{\partial x} \frac{\delta_{n, n_{2}-n_{1}} e^{i n_{2}\left(\omega_{0}+S x\right) \tau}}{\varepsilon\left[-i n_{2}\left(\omega_{0}+S x\right), n_{2}\right] \varepsilon\left[-i\left(n-n_{2}\right)\left(\omega_{0}+S x\right), n-n_{2}\right]} \\
& +\frac{\partial}{\partial x} \frac{\partial f_{0}(x)}{\partial x} \frac{\delta_{n, n_{2}-n_{1}} e^{-i\left(n+n_{1}\right)\left(\omega_{0}+S x\right) \tau}}{\varepsilon\left[i n_{1}\left(\omega_{0}+S x\right),-n_{1}\right] \varepsilon\left[-i\left(n+n_{1}\right)\left(\omega_{0}+S x\right), n+n_{1}\right]} \\
& \left.+\frac{\partial}{\partial x} \frac{\partial f_{0}(x)}{\partial x} \frac{\delta_{n, n_{1}-n_{2}} e^{i\left(n-n_{1}\right)\left(\omega_{0}+S x\right) \tau}}{\varepsilon\left[-i n_{1}\left(\omega_{0}+S x\right), n_{1}\right] \varepsilon\left[-i\left(n-n_{1}\right)\left(\omega_{0}+S x\right), n-n_{1}\right]}\right] .
\end{aligned}
$$

Upon substituting Eq. (53) for $F_{n}^{(2)}$ into Eq. (51) and summing over $n$ according to Eq. (50) we obtain the following expression for the second-order perturbation in the distribution function:

$$
\begin{aligned}
& f^{(2)}(x, \theta, t)=-\frac{q^{2} \omega_{0}^{2} N_{0}}{8 \pi^{3}}\left(\frac{\eta}{\beta_{0}^{2}} \frac{\omega_{0}}{\gamma_{0} M c^{2} S}\right)^{24 \pi i V_{1} V_{2} \pi^{2}} \frac{e^{i \Delta n \theta-i \Delta n\left(\omega_{0}+S x\right)}}{\omega_{0}^{2}} \frac{\partial}{\varepsilon\left[-i \Delta n\left(\omega_{0}+S x\right), \Delta n\right]} \frac{\partial}{\partial x} \\
& \times\left[\frac{\partial f_{0}(x)}{\partial x} \frac{e^{i n_{2}\left(\omega_{0}+S x\right) \tau}}{\varepsilon\left[-i n_{2}\left(\omega_{0}+S x\right), n_{2}\right] \varepsilon\left[i n_{1}\left(\omega_{0}+S x\right), n_{1}\right]} i \Delta n\left(\omega_{0}+S x\right)\right. \\
& \left.\times \int_{0}^{\infty} d t^{\prime} e^{-i \Delta n\left(\omega_{0}+S x\right) t^{\prime}+\left\{\left[D_{v}(\Delta n)^{2}\right] /\left(3 R^{2}\right)\right\}\left[\left(t-t^{\prime}\right)^{3}-t^{3}\right]}\right]+ \text { c.c. }
\end{aligned}
$$

The second-order perturbed current $I^{(2)}\left(\theta, t, D_{v} \neq 0\right)$ with diffusion corresponding to $f^{(2)}(x, \theta, t)$ is obtained as follows:

$$
\begin{aligned}
& I^{(2)}\left(\theta, t, D_{v} \neq 0\right)=\frac{q \omega_{0}}{2 \pi} \int_{-\infty}^{\infty} d x f^{(2)}(x, \theta, t) \\
& =-\frac{q^{3} \omega_{0}^{3} N_{0}}{16 \pi^{4}}\left(\frac{\eta}{\beta_{0}^{2}} \frac{\omega_{0}}{\gamma_{0} M c^{2} S}\right)^{24 \pi i V_{1} V_{2} \pi^{2}} \int_{-\infty}^{\infty} d x \frac{e^{i \Delta n \theta-i \Delta n\left(\omega_{0}+S x\right) t}}{\varepsilon\left[-i \Delta n\left(\omega_{0}+S x\right), \Delta n\right]} \frac{\partial}{\partial x} \\
& \times\left[\frac{\partial f_{0}(x)}{\partial x} \frac{e^{i n_{2}\left(\omega_{0}+S x\right) \tau} H\left(x, t, D_{v}\right)}{\varepsilon\left[-i n_{2}\left(\omega_{0}+S x\right), n_{2}\right] \varepsilon\left[i n_{1}\left(\omega_{0}+S x\right), n_{1}\right]}\right]+\text { c.c., }
\end{aligned}
$$

where we introduced the function $H\left(x, t, D_{v}\right)$ which now accounts for the contribution of the diffusion to the echo current,

$$
H\left(x, t, D_{v}\right)=-\int_{0}^{\infty} d t^{\prime} \frac{d}{d t^{\prime}}\left(e^{-i \Delta n\left(\omega_{0}+S x\right) t^{\prime}}\right) e^{\left[(\Delta n)^{2} /\left(3 R^{2}\right)\right] D_{v}\left[\left(t-t^{\prime}\right)^{3}-t^{3}\right]} .
$$

From Eq. (56) we see that the effect of diffusion on the longitudinal ballistic or collective echo amplitudes is to modify the amplitude by the contribution from the function $H\left(x, t, D_{v}\right)$. In the special case of diffusion free 
longitudinal echoes $D_{v}=0$, the function $H\left(x, t, D_{v}\right)$ becomes unity, and Eq. (55) becomes identical to Eq. (23). For the case of weak diffusion which we are interested in, the main contribution of the exponential term containing $D_{v}$ comes from the $t^{\prime}$ values close to $t$ or $t_{\text {echo }}=$ $\left[\left(n_{2}\right) /(\Delta n)\right] \tau$. Accordingly, the function $H\left(x, t, D_{v}\right)$ might be written as follows:

$$
H\left(t, D_{v}\right) \approx e^{\left[(\Delta n)^{2} /\left(3 R^{2}\right)\right] D_{v}\left\{\left[t-\left(n_{2} / \Delta n\right) \tau\right]^{3}-t^{3}\right\}} .
$$

For the case of weak diffusion, the echo current at harmonic $\Delta n$ becomes

$$
\begin{aligned}
I_{\Delta n}\left(t, D_{v} \neq 0\right) \approx & I_{\Delta n}\left(t, D_{v}=0\right) \\
& \times e^{\left[(\Delta n)^{2} /\left(3 R^{2}\right)\right] D_{v}\left\{\left[t-\left(n_{2} / \Delta n\right) \tau\right]^{3}-t^{3}\right\}} \\
\approx & I_{\Delta n}\left(t, D_{v}=0\right) e^{-\left[(\Delta n)^{2} /\left(3 R^{2}\right)\right] D_{v} t_{\text {echo }}^{3}}
\end{aligned}
$$

where $I_{\Delta n}\left(t, D_{v}=0\right)$ means the ballistic echo amplitude in Eq. (29) for $\varepsilon=1$ or the collective echo amplitude in Eq. (37) for $\varepsilon \neq 1$.

The self-consistent expression in Eq. (55) accounts for the effect of arbitrary diffusion on the longitudinal ballistic and collective beam echo. The approximate result in Eq. (58) shows that the space-charge and diffusion effects can be decoupled for weak diffusion, a result which recovers previously reported results concerning the effect of weak diffusion on plasma wave echoes [4] as well as on longitudinal ballistic beam echoes $[13,17]$.

As long as the effect of diffusion is weak, we conclude that the effect of weak diffusion is to modify in the same manner the ballistic and the collective echo amplitudes, namely, a modification by an exponential factor that varies with the third power of the echo time. In the case of arbitrary diffusion, the function $H\left(x, t, D_{v}\right)$ should be calculated with care and the decoupling of the spacecharge and diffusion effects is no more possible.

\section{NUMERICAL ANALYSIS}

In the present section we compare the analytic results obtained in Sec. III with numerical simulations. The choice of the numerical method is not trivial because already weak diffusion will smear out the echo effect. In particular, when coupled self-consistently to the spacecharge fields, standard particle tracking codes suffer from artificial diffusion due to simulation noise [24]. Here we employ a direct, grid-based solver (Vlasov-FokkerPlanck simulation) for Eqs. (6) and (7) in longitudinal $(z, v)$ phase space. $z=R\left(\theta-\theta_{0}\right)$ is the longitudinal coordinate in the reference frame comoving with the synchronous particle.

Details of the solver and its application to collective phenomena in longitudinal beam dynamics have been described in Ref. [25]. In all example cases, we use the following beam parameters, typical for the GSI cooler storage ring: $E_{k}=340 \mathrm{MeV} / \mathrm{u}$ is the kinetic energy per nucleon, $\gamma_{t}=3$ is the transition energy, $L=108 \mathrm{~m}$ is the ring circumference, $A=12$ and $Z=6$ are the mass and charge numbers of the carbon ions, respectively, and $\sigma_{p}^{\mathrm{FWHM}}=1.1 \times 10^{-5}$ is the momentum spread of the Gaussian momentum distribution function. The harmonics of the two rf kicks are chosen as $n_{1}=9$ and $n_{2}=10$, the rf voltage of both kicks is $50 \mathrm{mV}$, and their duration is $1 \mathrm{~ms}$. The relative current perturbation induced by the $\mathrm{rf}$ kicks remains below $1 \%$.

\section{A. Ballistic echo}

In Fig. 1 we plot the time evolution of the relative echo amplitudes defined as

$$
\frac{\delta I_{\Delta n}(t)}{I_{0}}=\frac{I_{\Delta n}(t)-I_{0}}{I_{0}}
$$

obtained from the ballistic echo theory in Eq. (29) and from the Vlasov simulation. The delay time between the two $\mathrm{rf}$ kicks is chosen as $\tau=0.1 \mathrm{~s}$. The echo is centered around $t=10 \tau$. This is consistent with the predicted echo time $t_{\text {echo }}=n_{2} \tau / \Delta n$. The agreement between the echo amplitudes obtained from theory and simulation is very good. Both show the same double peak structure with unequal heights. The peak echo amplitudes obtained from the simulation slightly underestimate the theoretically predicted peak amplitudes.

\section{B. Collective echo}

In Fig. 2 we plot the time evolution of collective echo amplitudes for $U=U_{\Delta n}=U_{n_{1}}=U_{n_{2}}=1$ resulting from the analytic theory Eq. (45) and from the Vlasov simulation together with the ballistic echo amplitude $(U=0)$. In contrast to the analytic result for $U=1$, the echo amplitude obtained from the simulation oscillates with approximately twice the space-charge induced frequency shift $\Delta \omega=\omega(\Delta n)-n \omega_{0}= \pm n S \sqrt{U}$. In our

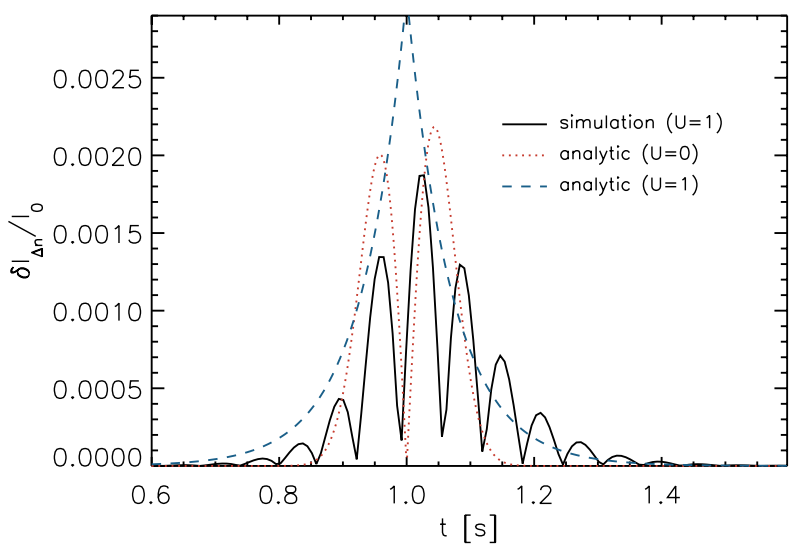

FIG. 2. (Color) Comparison of the echo amplitude versus time obtained from the simulation (solid line), the analytic ballistic echo theory (red dotted line), and from the analytic collective echo theory (blue dashed line) for $U=1$ and $\tau=0.1 \mathrm{~s}$. 
simulation as well as in a real storage ring experiment using rf kicks we automatically excite both space-charge modes, the backward running ("slow") mode and the forward running ("fast") mode. The superposition of the resulting slow (-) and fast $(+)$ echoes leads to the total echo amplitude

$$
I_{\Delta n}(t)=2 I_{\Delta n}^{ \pm}(t) \sin \left(2|\Delta \omega| t+\gamma_{\Delta n}\right)
$$

where the phase $\gamma_{\Delta n}$ is undetermined. On the other hand, the analytic result in Eq. (37) or (45) corresponds to only one of the two possible echo amplitudes $I_{\Delta n}^{ \pm}$. Therefore, we divided the simulation data by a factor of 2 in Fig. 2 and used the amplitudes at times $2|\Delta \omega| t+\gamma_{\Delta n}=0$ (the local maxima) only when comparing with analytic results. It is worth noting that very similar oscillation patterns, probably induced by ring impedances, were observed during echo experiments in the SPS ([26]).

In Fig. 2 we see that the simulation gives a slightly asymmetric echo about $t_{\text {echo }}=1 \mathrm{~s}$, whereas the analytic result is symmetric. The maximum echo amplitude found from Eq. (47) overestimates the maximum amplitude obtained from the simulation. However, the damping rates of the simulated and the analytic echo amplitudes are in good agreement. The increased width of the collective echo amplitude relative to the ballistic case can also be observed in the simulation. With increasing spacecharge parameter $U$ the damping rates and the peak amplitudes around $t=t_{\text {echo }}$ found from the simulation are underestimated by Eq. (45). This can be seen from Fig. 3 for $U=1.5$ and Fig. 4 for $U=2$.

It is interesting to point out that the maximum echo amplitude found from the simulation is essentially independent of the space-charge parameter $U$. This is in contrast to the analytic theory where the maximum echo amplitude [Eq. (47)] decreases exponentially for large space-charge parameters $U$. We verified carefully

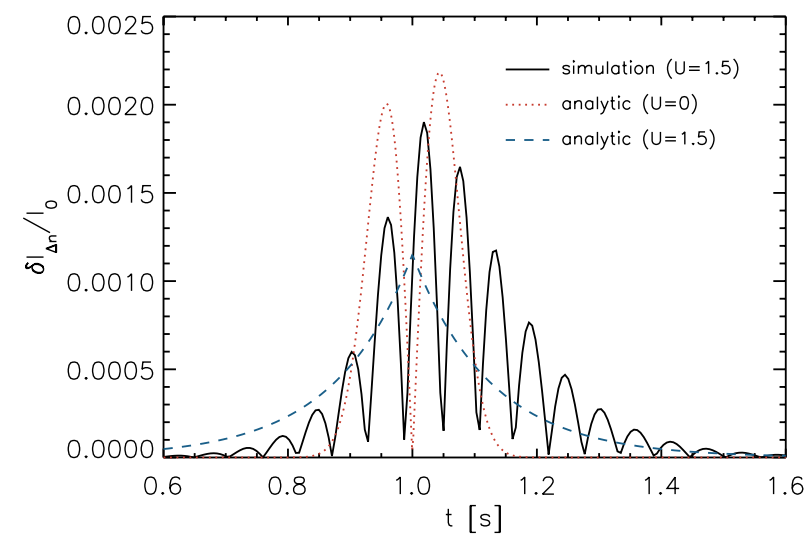

FIG. 3. (Color) Comparison of the echo amplitude versus time obtained from the simulation (solid line), the analytic ballistic echo theory (red dotted line), and from the analytic collective echo theory (blue dashed line) for $U=1.5$ and $\tau=0.1 \mathrm{~s}$.

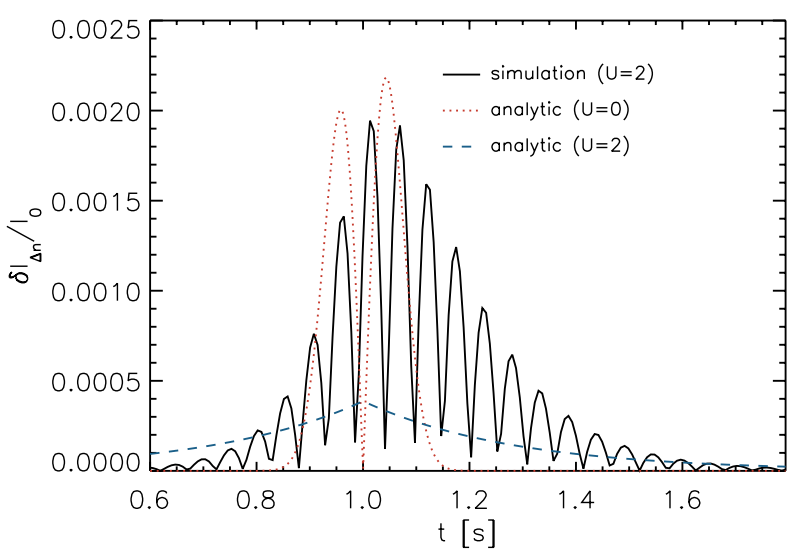

FIG. 4. (Color) Comparison of the echo amplitude versus time obtained from the simulation (solid line), the analytic ballistic echo theory (red dotted line), and from the analytic collective echo theory (blue dashed line) for $U=2$ and $\tau=0.1 \mathrm{~s}$.

that the simulation results around $t_{\text {echo }}$ do not change significantly when changing various simulation parameters, such as the grid spacing or the time step. Therefore, we conclude that our analytic theory cannot be used to accurately predict the time evolution of the collective echo amplitudes for times close to $t_{\text {echo }}$. The detailed reasons for this limitation of our analytic theory are still under investigation. One possible explanation is that with increasing $U$ the Landau damping times increase and at some point cannot be regarded as short relative to the delay time $\tau$.

For $U<1$ the transition to the ballistic, double peaked amplitude can be observed in the simulation. In this transition regime $0<U<1$, Eq. (45) is not valid. The simulation result for $U=0.5$ in Fig. 5 shows the reappearance of the two ballistic echo peaks together with

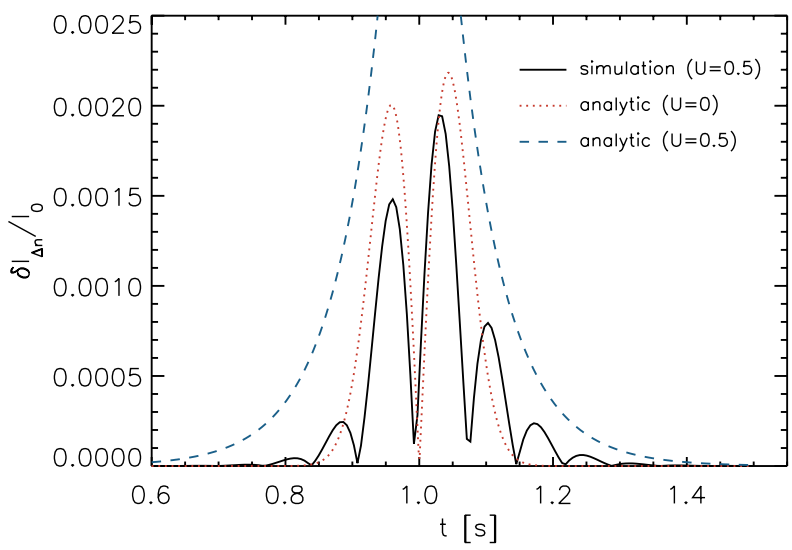

FIG. 5. (Color) Comparison of the echo amplitude versus time obtained from the simulation (solid line), the analytic ballistic echo theory (red dotted line), and from the analytic collective echo theory (blue dashed line) for $U=0.5$ and $\tau=0.1 \mathrm{~s}$. 
"tails" that exhibit a damping behavior similar to Eq. (45).

\section{Effect of diffusion}

We will now turn to studies including the effect of a small diffusion coefficient $D_{v}$ in our Vlasov-FokkerPlanck simulation code. The simulation results for the maximum echo amplitude as a function of the echo time $t_{\text {echo }}=n_{2} \tau / \Delta n$ can be compared to Eq. (58) using the following form:

$$
I_{\Delta n}^{\max }\left(t_{\text {echo }}\right)=I_{\Delta n}^{\max }\left(t_{\text {echo }}, D_{v}=0\right) e^{-(1 / 3)\left(D_{v} / R^{2}\right)(\Delta n)^{2} t_{\text {echo }}^{3}} .
$$

For ballistic echoes, the amplitude $I_{\Delta n}^{\max }\left(t_{\text {echo }}, D_{v}=0\right)$ can be determined analytically from Eq. (33). In the case of collective echoes we use an amplitude fit factor in Eq. (47). This is necessary because the absolute value of the maximum echo amplitude obtained from Eq. (37) is not in agreement with the simulation (see Sec. IV B).

Figure 6 shows the maximum echo amplitude obtained from the simulation and from the theory for $D_{v}=$ $200 \mathrm{~m}^{2} / \mathrm{s}^{3}$. In the ballistic echo case $(U=0)$, the simulation result agrees very well with the analytic result. Only around $t_{\mathrm{echo}} \approx 2 \mathrm{~s}$ a slight derivation of the simulated amplitude from the calculated one is observed. Also in the collective case $(U=1)$ good agreement between simulation and Eq. (61) (with an amplitude fitting parameter) is observed, with a slight deviation around $t_{\text {echo }} \approx 2 \mathrm{~s}$ and for very low $t_{\text {echo }}$. The same holds for the case $U=2$ not shown in the figure.

From this we can conclude that the effect of weak diffusion on the echo amplitude is independent of collective effects. For ballistic and collective coasting beam echoes, the effect of diffusion can be cast into the same simple multiplicative factor [Eq. (61)].

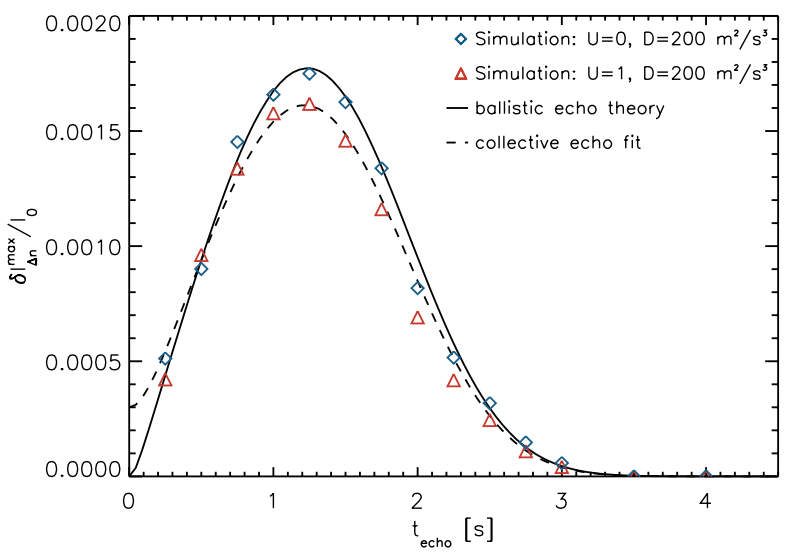

FIG. 6. (Color) Comparison of the maximum echo amplitude versus the echo time $t_{\text {echo }}$ obtained from the simulation $(U=0$ blue diamonds, $U=1$ red triangles), from the ballistic echo theory for finite $D$ (solid line), and from the amplitude fit to the collective echo theory (dashed line).

\section{Effect of numerical diffusion}

Grid-based Vlasov solvers suffer from numerical diffusion due to the interpolation on the grid that is required each time step. For the interpretation of simulation studies of collisionless, long-term kinetic phenomena it is important to know the amount of numerical diffusion beforehand. Another example is simulation studies of weak "physical" diffusion effects, such as those performed in the previous Sec. IVC. These studies require that the physical $D$ is much larger than the numerical $D$ introduced by the Vlasov solver. In order to determine the corresponding artificial velocity diffusion rate $\tau_{f}$ due to numerical errors in Vlasov simulations, or in any other kind of particle beam simulation scheme, one can try to fit the obtained maximum echo amplitude as a function of the echo time $t_{\text {echo }}$ to Eq. (61).

Figure 7 shows the maximum echo amplitude for $U=$ $0, U=1$, and $U=2$ as a function of the echo time $t_{\text {echo }}$ following from the Vlasov simulation (zero physical diffusion $D=0$ ). For $t_{\text {echo }} \lesssim 1.5 \mathrm{~s}$ the maximum echo amplitude is not affected by numerical diffusion. This shows that the effect of numerical diffusion on the simulation results presented in Figs. 1-5 for $t_{\text {echo }}=1 \mathrm{~s}$ can be neglected. For $t_{\text {echo }}>1.5 \mathrm{~s}$ numerical diffusion reduces the echo amplitudes. The simulation results agree very well with Eq. (61) if $D_{v}$ is used as a fitting parameter and the maximum echo amplitudes $I_{\Delta n}^{\max }\left(\tau, D_{v}=0\right)$ are determined from Eq. (33). For the ballistic echo a numerical diffusion coefficient $D_{v} \approx 18 \mathrm{~m}^{2} / \mathrm{s}^{3} \quad\left(\tau_{f} \approx 9000 \mathrm{~s}\right)$ is found. In the case of collective echoes the effect of numerical diffusion is found to be stronger, namely, $D_{v} \approx$ $35 \mathrm{~m}^{2} / \mathrm{s}^{3}\left(\tau_{f} \approx 4500 \mathrm{~s}\right)$. All simulations presented in this paper were performed with the same grid spacings and advection scheme. About a 100 grid points per rms

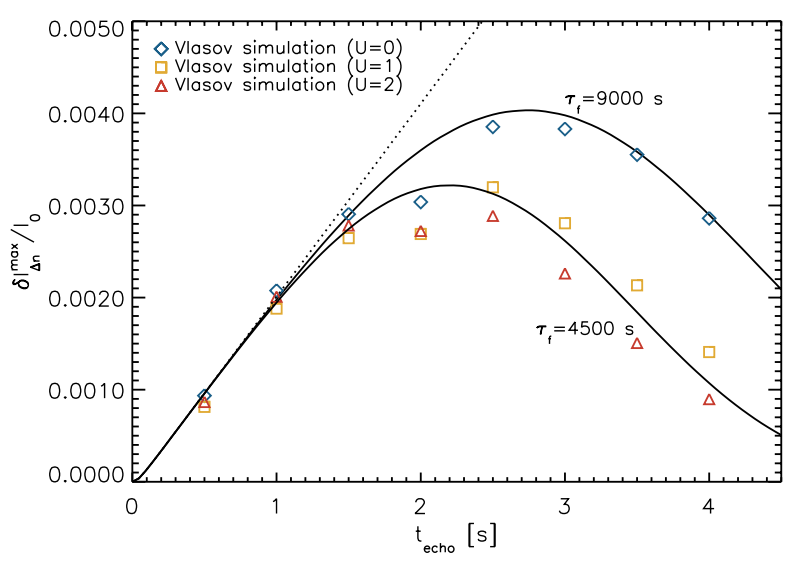

FIG. 7. (Color) Comparison of the maximum echo amplitude versus the echo time $t_{\text {echo }}$ obtained from the simulation $(U=0$ blue diamonds, $U=1$ orange squares, $U=2$ red triangles), from the ballistic echo theory for zero (dotted line), finite $D$ (solid line), and from the amplitude fit to the collective echo theory (dashed line). 
velocity width (total 500 points along the $v$ axis) and 512 grid points along the $z$ axis $(50$ grid points per harmonic $n_{2}=10$ ) were used. Preliminary results indicate that numerical diffusion scales according to $D_{v} \sim 1 / N_{v}$, with the number grid points in velocity space $N_{v}$.

For the advection of the distribution function on the grid the flux balance method [27] was used. A discussion of the different advections schemes for Vlasov solvers is given in Ref. [28]. The fact that numerical diffusion in collective echo simulations is about twice as strong as in ballistic echo simulations is likely due to the aliasing effect induced by the self-consistent coupling to the space-charge field that requires Fourier transformations each time step. A detailed study of numerical diffusion induced by different advection schemes together with a comparison of direct and particle methods is the topic of ongoing work.

\section{CONCLUSIONS}

In the framework of the nonlinear Vlasov theory the effect of space charge and diffusion on longitudinal echoes in coasting particle beams has been studied. Using the Vlasov equation for longitudinal beam dynamics and a second-order perturbation analysis, closed form analytical expressions for the time evolution of the ballistic and collective beam echo amplitudes have been obtained [see Eqs. (29), (37), and (58)]. The main result of the presented self-consistent theory is the calculation of the expressions for the collective echo amplitudes, a case that had not been studied before. The effect of diffusion on the echo amplitude is found to affect the ballistic and collective echo amplitudes in the same manner, namely, by an exponential decay factor varying with the time $t$ to the power of 3 . Vlasov simulations were found in excellent agreement with the ballistic echo theory, also if the effect of weak diffusion is added. In the case of collective echoes the simulation shows that the superposition of the slow and the fast echo waves leads to an oscillating echo amplitude. We found that our Vlasov theory cannot accurately predict the maximum echo amplitudes observed in the simulation for space-charge dominated beams. Nevertheless, the exponential damping of the echo amplitudes at both sides of the echo time $t_{\text {echo }}$ and the single peaked shape of the echo amplitude are both observed in the simulation. The simulations demonstrated that weak diffusion reduces the ballistic and the collective echo amplitudes by the same multiplicative factor. This decoupling of collective and diffusion effects will greatly simplify the interpretation of upcoming echo experiments in spacecharge dominated coasting beams. As an interesting side application we used echoes in order to determine the numerical diffusion in our simulation scheme. This could be a powerful method to quantify the long-time accuracy of self-consistent particle simulation schemes. The present work will be the basis for the measurement of diffusion rates in stored, electron cooled ion beams in storage rings.

\section{ACKNOWLEDGMENTS}

A. A.-K. would like to thank GSI-Darmstadt for the financial support of his stay there in 2001/2002. He would also like to thank the Council of Scientific Research at Yarmouk University, Irbid, Jordan, for partial support.

*Permanent address: Department of Physics, Faculty of Science, Yarmouk University, Irbid, Jordan.

${ }^{\dagger}$ Corresponding author.

Email address: O.Boine-Frankenheim@gsi.de

[1] R.W. Gould, T. M. O'Neil, and J. H. Malmberg, Phys. Rev. Lett. 19, 219 (1967).

[2] T. M. O'Neil and R.W. Gould, Phys. Fluids 11, 1 (1968).

[3] J. H. Malmberg, C. B. Wharton, R.W. Gould, and T. M. O’Neil, Phys. Rev. Lett. 20, 95 (1968).

[4] C. H. Su and C. Oberman, Phys. Rev. Lett. 20, 427 (1968).

[5] T. M. O’Neil, Phys. Fluids 11, 2420 (1968).

[6] T. H. Jensen, J. H. Malmberg, and T. M. O'Neil, Phys. Fluids 12, 1728 (1969).

[7] M. Porkolab and R. P. H. Chang, Rev. Mod. Phys. 50, 745 (1978).

[8] B. Ripin and R. Pechacek, Phys. Rev. Lett. 24, 1330 (1970).

[9] Ronald C. Davidson, Methods in Nonlinear Plasma Theory (Academic Press, New York, 1972), pp. 89-100.

[10] G.V. Stupakov, Superconducting Supercollider Laboratory Report No. SSCL-579, 1992.

[11] G.V. Stupakov and S. Kauffmann, Superconducting Supercollider Laboratory Report No. SSCL-587, 1992.

[12] G.V. Stupakov and A.W. Chao, in Proceedings of the 1997 Particle Accelerator Conference, Vancouver, B.C., Canada (IEEE, Piscataway, NJ, 1997), pp. 1834-1836.

[13] O. Brüning, CERN Report No. SL/95-83 (AP), 1995.

[14] P. L. Colestock, L. K. Spentzouris, and J.-F. Ostiguy, in Proceedings of the 1995 Particle Accelerator Conference, Dallas, Texas (IEEE, Piscataway, NJ, 1995), pp. 2757-2761.

[15] O. Brüning, T. Linnecar, F. Ruggiero, W. Scandale, E. Shaposhnikova, and D. Stellfeld, in Proceedings of the EPAC 96, Barcelona, Spain (EPAC/CERN, Geneva, 1996).

[16] L. K. Spentzouris, J.-F. Ostiguy, and P. L. Colestock, Phys. Rev. Lett. 76, 620 (1996).

[17] A. Wu Chao, in Proceedings of the U.S. Particle Accelerator School, Lecture Notes on Topics in Accelerator Physics [SLAC, Stanford (unpublished)].

[18] P. L. Colestock, L. K. Spentzouris, and F. Ostiguy, in Proceedings of the 1995 Particle Accelerator Conference, Dallas, Texas (Ref. [14]), p. 2757.

[19] A. Al-Khateeb, O. Boine-Frankenheim, I. Hofmann, and G. Rumolo, Phys. Rev. E 63, 026503 (2001).

[20] S. A. Bogacz and K-Y. Ng, Phys. Rev. D 36, 1538 (1987). 
[21] Yongho Chin and Kaoru Yokoya, Phys. Rev. D 28, 2141 (1983).

[22] M. Reiser, Theory and Design of Charged Particle Beams (John Wiley \& Sons, Inc., New York, 1994).

[23] K. L. Spenzouris, P. L. Colestock, and C. Bhat, in Proceedings of the 1999 Particle Accelerator Conference, New York (IEEE, Piscataway, NJ, 1999), pp. $114-117$.

[24] C. K. Birdsall and A. B. Langdon, Plasma Physics via Computer Simulation (IOP Publishing, Bristol, 1995), p. 266.
[25] O. Boine-Frankenheim, I. Hofmann, and G. Rumolo, Phys. Rev. Lett. 82, 3256 (1999); O. BoineFrankenheim and I. Hofmann, Phys. Rev. ST Accel. Beams 3, 104202 (2000).

[26] O. Brüning, T. Linnecar, F. Ruggiero, W. Scandale, E. Shaposhnikova, and D. Stellfield, CERN SL-MD Note No. 206, 1996.

[27] E. Fijalkow, Comput. Phys. Commun. 116, 319 (1999).

[28] T. D. Arber and R. G. L. Vann, J. Comput. Phys. 180, 339 (2002). 